\title{
Editorial
}

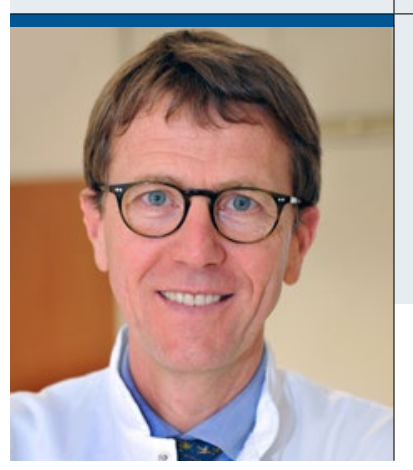

„Das Handwerkszeug zur Wiedereröffnung chronischer Gefäßverschlüsse hat sich stark gewandelt. Innovative Instrumente und spezielle Techniken haben eine dramatische Steigerung der Erfolgsrate bewirkt."

\section{Neue Herausforderungen der interventionellen Koronartherapie}

D ie interventionelle Therapie der koronaren Herzerkrankung hat in den letzten Jahren ihren Erfolgskurs fortgesetzt. Die Möglichkeiten der kompletten Revaskularisierung mittels interventioneller Verfahren haben rasant zugenommen und längst ist die PCI nicht nur unkomplizierten Koronarläsionen vorbehalten, sondern auch bei komplexer Koronaranatomie überaus erfolgreich.

In dieser Ausgabe von CardioVasc werden drei aktuelle Herausforderungen der interventionellen Koronartherapie kritisch bewertet: Hauptstammstenose, chronische Gefäßverschlüsse und bioresorbierbare Stents. Vergleichende Untersuchungen konnten zeigen, dass auch bei Stenosen des Hauptstammes der linken Kranzarterie eine interventionelle Therapie möglich und erfolgreich ist, wenn die richtigen Patienten dem minimalinvasiven Verfahren zugeführt werden. In der Tat ist es so, dass Patienten mit unkomplizierter Hauptstammstenose und niedrigem SYNTAX-Score von der Intervention stärker als von der operativen Revaskularisierung profitieren.

Während sich die PCI der Hauptstammstenose dem klassischen Instrumentarium des Interventionalisten bedient, hat sich das Handwerkszeug zur Wiedereröffnung chronischer Gefäßverschlüsse stark gewandelt. Drähte mit unterschiedlicher Härte, Beschichtung und Auflagefläche der Drahtspitze erlauben es antegrad, retrograd oder subintimal Anschluss an das offene Gefäßlumen zu gewinnen. Neben weiteren innovativen Instrumenten kommen spezielle Techniken zum Einsatz, die eine dramatische Steigerung der Erfolgsrate bewirkt haben.

Die letzten beiden Jahre haben zudem umfassende Studienergebnisse mit Koronarscaffolds, also resorbierbaren Stents, erbracht, die eine sorgfältige Bewertung und Risikoabwägung im Vergleich zu den Drug Eluting Stents der neuesten Generation notwendig machen. $\mathrm{Ob}$ sich die aktuell verfügbaren Koronarscaffolds gegenüber konventionellen Stents vorteilhaft einsetzen lassen, wird von den Kollegen Dr. Cassese und Dr. Kufner im Detail beantwortet. Dr. Fusaro ist ein ausgewiesener Experte für die Rekanalisation von chronischen Koronarverschlüssen und konnte für einen Übersichtsartikel zu diesem Thema gewonnen werden. Schließlich sind Dr. Wiebe und Dr. Byrne nicht nur in hochrangigen wissenschaftlichen Journalen, sondern auch in diesen Heft von CardioVasc zu den aktuellen Möglichkeiten der Koronarintervention im Hauptstamm der linken Kranzarterie zu lesen.

Ich bin sehr froh, dass sich erneut renommierte Autoren für praxisrelevante und gut illustrierte Beiträge für dieses Heft von CardioVasc gewinnen ließen. Ich wünsche Ihnen viel Freude und nutzbringende Informationen beim Studium dieses Heftes.

\section{Mit herzlichen Grüßen \\ Ihr}

Prof. Dr. Heribert Schunkert

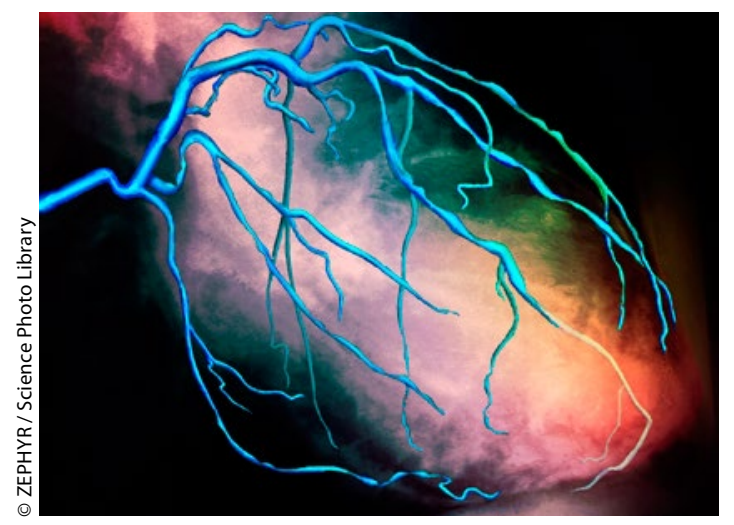

Bei der KHK ist die $\mathrm{PCl}$ auch bei komplexer Anatomie sehr erfolgreich, auch die komplette Rekanalisation chronischer Koronarverschlüsse mittels interventioneller Verfahren ist viel versprechend. 